\title{
SITUAÇÃO NUTRICIONAL E ALIMENTAR DE PORTADORES DE MALÁRIA RESIDENTES NO AMAZONAS - BRASIL
}

\author{
Fernando Hélio ALENCAR', Lucia Kiyoko Ozaki YUYAMA', \\ Miriam Elenit Lima GUTIERREZ ${ }^{2}$
}

\begin{abstract}
RESUMO - Foram estudados 268 indivíduos (198 homens e 70 mulheres), residentes no Amazonas, com resultados hemoscópicos positivos de malária, diagnosticados pela Fundação Nacional de Saúde - FNS/Manaus. A avaliação antropométrica foi universal, envolvendo as medidas de Peso, Altura, Índice de Massa Corpórea (IMC), Prega Cutânea Triciptal (PCT), Circunferência Braquial (CB) e Circunferência Muscular do Braço (CMB). O universo estudado foi ainda subamostrado para avaliação Hematológica, Bioquímica (121 indivíduos) e Parasitológica (55 individuos). A análise dos resultados evidencia que apesar da presença da malária e das precárias condiçòes de vida dos doentes, constatou-se que o Peso, Altura, IMC, CB e CMB apresentaram valores médios considerados normais, excetuando-se os resultados da PCT que foram inferiores aos padrōes considerados de normalidade. Os resultados de Hematócrito, Hemoglobina, Glicose, Ferro sérico e Triglicerídeos, apresentaram-se dentro dos limites de normalidade, estando a Proteina Total no limite inferior recomendado, e, o HDL colesterol abaixo do limite de normalidade. $\mathrm{O}$ inquérito de consumo alimentar revelou que a populaçăo estudada teve acesso a uma dieta bem estruturada, sendo freqüente o consumo de alimentos construtores (peixe, carnes e ovos), abundantes os energéticos: arroz, pão, gordura vegetal (óleos), e, em menor quantidade os reguladores (verduras e frutas). Como prováveis agravantes do estado nutricional dos portadores de malária, constatou-se alto índice de parasitose gastrointestinal, predominando o poliparasitismo, precárias condições de habitação, baixo nível de escolaridade, baixa renda, alto consumo de cigarro e álcool.
\end{abstract}

Palavras-chave: Estado nutricional; Malária; Plasmodium.

Nutritional and Dietary Status of Individuals With Malaria resident in Amazonas State-Brazil.

ABSTRACT - Two hundred sixty-eight subjects (198 men and 70 women) with blood-smears positive for malaria parasites, diagnosed at the Fundação Nacional de Saúde in Manaus were studied. The antthopometric evaluations of weight, height, body mass index, triceps skinfold thichness, brachial circunference and arm muscle circunference were performed an all subjects. Haematological and biochemical evaluations were performed for 121 individuals and coprological examination for parasites for 55 . Despite the malaria and precarious living conditions of the patients, the anthopometric mean values were normal with the exception of triceps skinfold thickness, which was below normal. Measures of haematocrit, haemoglobin, glicose, serum iron and triglycerides were within the normal range, with total protein at the lower limit of the recomended range, and HDL cholesterol below the limit of normality. The dietary investigation revealed that the study population had acess to a well balanced diet, with frequent consumption of proteinrich food (fish, meat and eggs), abundant sources of energy (rice, bread and vegetable oil) and smalles quantities of regulators (green vegetables and fruits). Among factors aggravating the nutritional status of the malaria patients, a high incidence of multiple gastrointestinal parasitosis, precarious living conditions, low educational status, low income and high consumption of tobacco and alcohol were detected.

Key-words: Status nutritional; Malaria; Plasmodium,

\footnotetext{
IINPA/CPCS. Caixa Posta 478. Manaus, AM. Brasil 969011-970

${ }^{2}$ Aluna do Curso de Pós Graduação em Ciências de alimentos da Universidade do Amazonas
} 


\section{INTRODUÇÃO}

Atualmente já são bem conhecidas as influências que determinados fatores exercem na gênese dos processos carenciais, principalmente, aqueles relacionados a população, pobreza e degradação ambiental, em cujo complexo etiológico interagem: redução do poder aquisitivo, subemprego, baixo nivel de escolaridade, precariedade de saneamento básico e crescimento demográfico exagerado, os quais favorecem ao aparecimento de processos infecciosos e parasitários (Alencar, 1988; OPAS, 1992; Batista, 1995; OMS,1992).

As carências nutricionais por insuficiência quantitativa, qualitativa ou decorrentes de doenças, têm um importante impacto sobre a suscetibilidade e resistência do hospedeiro, podendo interferir na competência funcional do sistema imunológico e comprometer o equilíbrio normalmente existente entre a "nutrição e imunidade"; contribuindo assim para a redução da resistência do hospedeiro, e, conseqüentemente, para instalaçâo de agentes infecciosos, distúrbios da capacidade física e mental (Beisel, 1980; OPAS, 1992;Paiva \& Campana,1992).

A malária é uma das doenças parasitárias que alcança extensas áreas no contexto mundial, particularmente, nas regiões tropicais e subtropicais, de prevalência endêmica nos países subdesenvolvidos, onde as condições sócio-econômicas, culturais e ambientais são favoráveis à manutenção e proliferação do mosquito transmissor. Nestas Regiões constatou-se alterações no padrão nutricional da população, primeiramente, em decorrência da redução da capacidade física do portador de malária em produzir o seu próprio alimento, bem como pela significativa alteração da capacidade fisiológica de aproveitamento dos alimentos, visto que a infecção malárica exerce intensa estimulação imunológica, devido a liberação prolongada de uma variedade de antígenos na circulação, cuja intensidade depende da espécie do Plasmodium (Ferraroni \& Speer, 1980;OPAS,1996).

A OMS (1993) considera a malária como o maior problema de saúde pública em muitos países, particularmente,

nos subdesenvolvidos, onde causa grande impacto sobre o desenvolvimento social e econômico, com altas taxas de morbidade e mortalidade. Estima-se que sua incidência seja da ordem de 300 a 500 milhões de casos clinicos por ano, sendo que mais de $90 \%$ destes casos ocorrem nos paises tropicais (Tauil, 1996).

No Brasil, a malária se constitui na mais expressiva das endemias. Incide fundamentalmente na região da Bacia Amazônica, incluindo os Estados do Acre, Amazonas, Roraima, Amapá, Rondônia, Pará, Mato Grosso, Tocantins e a região ocidental do Maranhão (Souza et al, 1997).

Nas últimas décadas, o município de Manaus tem sido palco de consideráveis surtos de malária, com influência significativa da área 
peri-urbana, determinada principalmente pelo $P$. falciparum. Em 1993, registrou-se o maior número de casos de malária na cidade (23.189 casos), dos quais, $58,8 \%$ foram consideradas autoctones. Alecrim (1995) e Albuquerque \& Mutis (1998), ressaltaram que os dois grandes focos disseminadores de malária em Manaus foram as áreas representadas pelos Bairros de Puraquequara e Tarumã.

Considerando-se então a magnitude dos problemas decorrentes da malária em nossa Região, bem como a lacuna de informações a respeito do comprometimento desta doença no estado nutricional do Amazônida, o presente estudo objetivou avaliar o estado nutricional e alimentar de individuos acometidos por malária residentes no Amazonas.

\section{MATERIAL E MÉTODOS}

O estudo foi realizado no Laboratório Central de Diagnóstico e Tratamento de Malária da Fundação Nacional de Saúde-FNS, Coordenadoria Regional-Manaus/Am. Foram estudados 268 indivíduos (198 homens e 70 mulheres), que se enquadraram nos seguintes critérios: faixa etária entre 15 a 50 anos, diagnóstico positivo de malária, tempo de residência não inferior a 5 anos na Amazônia e participação voluntária no estudo.

A avaliação antropométrica foi universal, utilizando as medidas de Peso e Altura, segundo as normas estabelecidas por Jelliffe (1968); Índice de Massa Corpórea - IMC (peso/altura $\left.^{2}\right)$ (Thomaz et al., 1976), espessura da Prega Cutânea Tricipital
(PCT) - utilizando-se o Skinfold Caliper Haltain; Circunferência Braquial (CB), por meio de fita milimétrica apropriada e Circunferência Muscular do Braço (CMB), calculada a partir da CB e PCT, de acordo com as recomendações de Frisancho (1974).

A investigação nutricional foi complementada ainda pela avaliação Hematológica e Bioquimica, envolvendo 121 amostras de sangue, obtidas por punção venosa em veia central do braço sendo determinados: Hemoglobina (método da cianometahemoglobina) (Van Kampen \& Zijltra, 1961); Hematócrito (técnica do microhematócrito); Glicose (método enzimático colorimétrico) (Trinder, 1969); Proteinas Totais (método de Biureto modificado); Ferro Sérico (método de Goodwin et al., 1966); HDL Colesterol (método enzimático de polietilenoglicol modificado) (Izzo et al., 1981) e Triglicerídeos (método enzimático colorimétrico) (Bucolo \& David, 1973). O inquérito alimentar, foi determinado pela técnica de freqüência de consumo alimentar, adotando-se a relação dos alimentos básicos que compõem o padrão alimentar da dieta regional de Manaus (Shrimpton \& Giugliano, 1979; Yuyama \& Cozzolino, 1995).

$\mathrm{Na}$ análise estatística dos dados foi utilizado o software Epi Info (Dean et al., 1990) e Systat (Wilkinson, 1990). Para observar a ocorrência de contraste entre as médias, foi utilizado o teste do $\chi^{2}$ e t - Student. As associações foram consideradas significativas quando $\mathrm{p}<0,05$. 


\section{RESULTADOS}

Dentre os 268 portadores de malária estudados houve maior representação para o sexo masculino $(73,9 \%)$; sendo mais acometidas as faixas etárias compreendidas entre 20 e 40 anos $(68,3 \%)$ e menos expressiva sua ocorrência entre os individuos com idade inferior a 20 anos $(14,9 \%)$ e acima dos 40 anos $16,8 \%$ (Tab. 1).
Na Tabela 2, constata-se um valor médio menor em Peso $(57,3 \mathrm{~kg})$, indice de Quetelet $\left(22,5 \mathrm{~kg} / \mathrm{m}^{2}\right)$, Circunferência Braquial $(26,6 \mathrm{~cm})$ e Circunferência Muscular do Braço $(23,9 \mathrm{~cm})$, para os pacientes menores de 20 anos $(\mathrm{p}<0,05)$. Para o sexo masculino registrou-se um valor médio maior tanto na estatura $(1,6 \mathrm{~m})$, como no Peso $(64,7 \mathrm{~kg})$, sendo menos expressiva a diferença nos valores

Tabela 1. Estatistica descritiva: Média (X), Desvio Padrão (DP), para as variáveis antropométricas segundo a idade, sexo, tipo de Plasmodium e $\mathrm{n}^{0}$ de malária em portadores residentes em Manaus(Am).

\begin{tabular}{|c|c|c|c|c|c|c|}
\hline $\begin{array}{c}\text { VARIÁV EIS } \\
\text { ANTROP OME TRICAS }\end{array}$ & $\begin{array}{l}\text { ESTATURA } \\
\text { (m) }\end{array}$ & $\begin{array}{c}\text { PESO } \\
(\mathrm{kg})\end{array}$ & $\begin{array}{c}\text { IMC } \\
(\mathrm{kg} / \mu 2)\end{array}$ & $\begin{array}{l}\text { PCT } \\
(\mathrm{mm})\end{array}$ & $C B(\mathrm{Cm})$ & $\begin{array}{l}\mathrm{CMB} \\
(\mathrm{Cm})\end{array}$ \\
\hline \multicolumn{7}{|l|}{ FAIXA ETÁ RIA } \\
\hline$<20$ anos & $1,6 \pm 0,1$ & $57,3 \pm 10,3$ & $22,5 \pm 3,3$ & $9,1 \pm 4,3$ & $26,6 \pm 3,3$ & $23,9 \pm 3,1$ \\
\hline 20.40 a nos & $1,6 \pm 0,1$ & $63,1 \pm 10,2$ & $24,2 \pm 3,2$ & $9,9 \pm 4,4$ & $28,6 \pm 2,9$ & $25,7 \pm 2,8$ \\
\hline$>40$ a nos & $1,6 \pm 0,1$ & $64,5 \pm 13,4$ & $24,3 \pm 4,2$ & $9,2 \pm 3,6$ & $28,8 \pm 3,1$ & $25 . \pm 2.7$ \\
\hline Média Geral & $1.6 \pm 0.1$ & $62,2 \pm 11,0$ & $23,8 \pm 3,5$ & $9,1 \pm 4,1$ & $28,2 \pm 3,1$ & $25,4 \pm 2,8$ \\
\hline$x^{2}$ & $p>0.05$ & $p>0,05$ & $p<0,05$ & $p>0.05$ & $p<0.05$ & $p<0,05$ \\
\hline \multicolumn{7}{|l|}{ SEXO } \\
\hline Masculino & $1,6 \pm 0,1$ & $64,7 \pm 10,4$ & $23,9 \pm 3,4$ & $7,6 \pm 3,2$ & $28,8 \pm 2,9$ & $26,4 \pm 2,6$ \\
\hline Feminino & $1,5 \pm 0,1$ & $56,1 \pm 10,6$ & $24,0 \pm 3,8$ & $13,1 \pm 4,1$ & $27,0 \pm 3,0$ & $23,2 \pm 2,7$ \\
\hline Mèdia Geral & $1,6 \pm 0,1$ & $60,4 \pm 10,5$ & $23,9 \pm 3,6$ & $10,4 \pm 3,7$ & $27,9 \pm 2,3$ & $24,8 \pm 2,6$ \\
\hline$x^{2}$ & $p<0,05$ & $p<0,05$ & $p>0.05$ & $p<0,05$ & $p<0,05$ & $p<0,05$ \\
\hline \multicolumn{7}{|l|}{ TOTAL DE PLASMODIUM } \\
\hline Vivax & $1,6 \pm 0,1$ & $62,2 \pm 11,4$ & $23,9 \pm 3,6$ & $9,1 \pm 4,3$ & $28.4 \pm 3,0$ & $25,6 \pm 3,1$ \\
\hline Falciparum & $1,6 \pm 0,1$ & 10,2 & $24,1 \pm 3,1$ & $9,0 \pm 4,0$ & $28,1 \pm 2,8$ & $25,4 \pm 2,7$ \\
\hline Média Geral & $1,6 \pm 0,1$ & $62,7 \pm 10,8$ & $24,0 \pm 3,4$ & $9,0 \pm 4,1$ & $28,3 \pm 3,0$ & $25,5 \pm 2,9$ \\
\hline$x^{2}$ & $p>0,05$ & $p>0.05$ & $p>0.05$ & $p>0,05$ & $p>0,05$ & $p>0,05$ \\
\hline \multicolumn{7}{|l|}{ NỦMERO DE MALÁ RIA } \\
\hline Uma vez & $1,6 \pm 0,1$ & $63,3 \pm 11,1$ & $24,1 \pm 3,8$ & $9,3 \pm 4,1$ & $28,4 \pm 3,1$ & $25,5 \pm 2,9$ \\
\hline Duas vezes & $1,6 \pm 0,1$ & $61,4 \pm 10,5$ & $23,8 \pm 3,2$ & $8,8 \pm 4,3$ & $28,4 \pm 3,1$ & $25,6 \pm 3,0$ \\
\hline Mais de Duas & $1,6 \pm 0,1$ & $61,9 \pm 11,1$ & $23,9 \pm 3,4$ & $8,9 \pm 4,3$ & $28,2 \pm 3,1$ & $25,5 \pm 2,9$ \\
\hline Média Geral & $1,6 \pm 0,1$ & $62,2 \pm 10,9$ & $23,9 \pm 3,5$ & $8,9 \pm 4,2$ & $28,3 \pm 3,1$ & $25,5 \pm 2,9$ \\
\hline$x^{2}$ & $p>0,05$ & $p>0,05$ & $p>0,05$ & $p>0,05$ & $p>0,05$ & $p>0,05$ \\
\hline
\end{tabular}


Tabela 2. Estatistica descritiva: Média (X), Desvio Padrão (DP), para as variáveis laboratoriais segundo a faixa etária, sexo e tipo de Plasmodium em portadores de malária residentes em Manaus(Am).

\begin{tabular}{|c|c|c|c|c|c|c|c|}
\hline $\begin{array}{l}\text { VARIÁVEIS } \\
\text { LABORATORIAIS }\end{array}$ & $\begin{array}{l}\text { HEMATO } \\
\text { CRIO }(\%)\end{array}$ & $\begin{array}{l}\text { HE MOGLOBINA } \\
\qquad(g / 100 \mathrm{dL})\end{array}$ & $\begin{array}{l}\text { GLICOSE } \\
\text { (mg/dil) }\end{array}$ & $\begin{array}{l}\text { PROTEINA } \\
\text { (gid) }\end{array}$ & $\begin{array}{c}\text { FERROSERACO } \\
\text { (山gAL) }\end{array}$ & $\begin{array}{l}\mathrm{HDC} \\
i \mathrm{mg} / \mathrm{dL}\end{array}$ & $\begin{array}{c}\text { TRKGLICERDEOS } \\
\text { (mgDL) }\end{array}$ \\
\hline \multicolumn{8}{|l|}{ FAIXA ETÁRIA } \\
\hline$<20$ anos & $40.7 \pm 5.0$ & $13,0=1.9$ & $80.5 \pm 12.1$ & $6,9 \pm 0,9$ & $775 \pm 315$ & $329 \pm 14.1$ & $101 \pm 52.8$ \\
\hline $20-40$ anos & $42.3 \pm 4.5$ & $13,3 \pm 1,7$ & $87.1 \pm 18.7$ & $6,9 \pm 08$ & $73,9 \pm 319$ & $29.1 \pm 202$ & $1172=78.5$ \\
\hline$>40$ anss & $41,7 \pm 5.7$ & $12,8 \pm 2,3$ & $90,9 \pm 21,9$ & $6,9 \pm 0,8$ & $78,6 \pm 44,7$ & $30,7 \pm 17,4$ & $155.5 \pm 111.9$ \\
\hline Media Geral & $41,9 \pm 48$ & $13,2=18$ & $86.4 \pm 18.3$ & $6.9=0.8$ & $76,0 \pm 34,7$ & $29.7 \pm 18 B$ & $120.8 \pm 81.3$ \\
\hline$x^{2}$ & $p>0.05$ & $p>0.05$ & $p<0,05$ & $p>0.05$ & $p<0.05$ & $p<0.05$ & $p>0,05$ \\
\hline \multicolumn{8}{|l|}{ SEXO } \\
\hline Masculino & $42.6 \pm 49$ & $13,4 \pm 1,9$ & $86,6 \pm 182$ & $6.9 \pm 0.8$ & $78,8 \pm 370$ & $28.8 \pm 17.5$ & $119.4 \pm 813$ \\
\hline Feminino & $39,7 \pm 3,9$ & $12,4 \pm 1.5$ & $85.9=188$ & $7,1 \pm 08$ & $67,1 \pm 24,7$ & $32,6 \pm 22,3$ & $125.2=82 A$ \\
\hline Mèdia Geral & $41,9 \pm 48$ & $13.2 \pm 18$ & $86,4 \pm 18,3$ & $6,9 \pm 0.8$ & $76,0 \pm 34,7$ & $29 . \pm 188$ & $1208 \pm 813$ \\
\hline$x^{2}$ & $D<0.05$ & $p<0,05$ & $p>0.05$ & $p>0.05$ & $p<0.05$ & $p<0.05$ & $p>0,05$ \\
\hline \multicolumn{8}{|l|}{ TOTAL DE PLASMODIUM } \\
\hline vivax & $41,9 \pm 4,9$ & $13.2 \pm 19$ & $85.4 \pm 19.0$ & $6,9 \pm 0.7$ & $75.6 \pm 372$ & $30.5 \pm 18.6$ & $120.9 \pm 80.2$ \\
\hline Falciparum & $41,8 \pm 4,6$ & $13.0 \pm 1.7$ & $89.1 \pm 16.5$ & $6,8 \pm 0.9$ & $76.3 \pm 282$ & $27.9 \pm 19.5$ & $115,0 \pm 79,4$ \\
\hline Mèdia Geral & $41,9 \pm 48$ & $13,2 \pm 1,8$ & $86,4 \pm 18,3$ & $6,9 \pm 0,8$ & $75.8 \pm 348$ & $298=18 B$ & $119.2 \pm 798$ \\
\hline$x^{2}$ & $p>0.05$ & $p>0.05$ & $p>0,05$ & $p>0,05$ & $p>0.05$ & $p>0.05$ & $p>0,05$ \\
\hline
\end{tabular}

médios no Índice de Quetelet $(23,9 \mathrm{~kg} /$ $\mathrm{m}^{2}$ ) para os homens e de $24,0 \mathrm{~kg} / \mathrm{m}^{2}$ para as mulheres. Entretanto, o valor médio da Prega Cutânea Tricipital foi, expressivamente, superior nas mulheres $(13,1 \mathrm{~mm})$ e $7,6 \mathrm{~mm}$ nos homens. Inversamente a Circunferência Braquial e Muscular do Braço, foram superiores nos homens $(28,6$ e $26,4 \mathrm{~cm})$, e de 27,0 e $23,2 \mathrm{~cm}$, nas mulheres. Constata-se ainda que a presença do Plasmodium vivax ou $P$. falciparum teve baixo poder em determinar alterações nas variáveis antropométricas, entretanto os pacientes que referiram dois, três e/ou mais episódios de malária, apresentaram valores médios de Peso e Índice de Quetelet, ralativamente inferiores aqueles que referiram apenas um episódio.

\section{Avaliação Hematológica e Bioquímica}

Constata-se pela Tabela 3 que os indivíduos acometidos de malária, independentemente da idade, apresentavam uma concentração média de HDL-Colesterol inferior ao padrão de normalidade. As demais 
variáveis encontravam-se dentro dos limites de normalidade. Confrontandose estes resultados em função do sexo, registra-se para os homens, valores expressivamente superiores na concentração de Ferro Sérico $(78,8$ $\mathrm{mg} / \mathrm{dL}$ ), médias mais baixas nas concentrações de HDL-Colesterol $(28,8 \mathrm{mg} / \mathrm{dL})$ e Triglicerídeos $(119,4$ $\mathrm{mg} / \mathrm{dl}$ ). Entretanto, não registramos alterações significativas nos valores médios das variáveis Hematológicas e Bioquímicas quando confrontadas com os tipos de Plasmodium (vivax e

Tabela 3. Freqüência do consumo de alimentos na dieta dos portadores de malária $(N=268)$, Manaus - Novembro de 1997.

\begin{tabular}{|c|c|c|c|c|c|c|c|c|c|}
\hline \multirow{3}{*}{$\begin{array}{l}\text { GRUPO } \\
\text { Alimentos }\end{array}$} & \multirow[t]{3}{*}{ TIPO } & \multicolumn{8}{|c|}{ FREQŪÊNCIA. } \\
\hline & & \multicolumn{2}{|c|}{ Diária } & \multicolumn{2}{|c|}{ Semanal } & \multicolumn{2}{|c|}{ Mensal } & \multicolumn{2}{|c|}{ Casual } \\
\hline & & $\mathrm{N}$ & $\%$ & $\mathrm{~N}$ & $\%$ & $\mathrm{~N}$ & $\%$ & $\mathrm{~N}$ & $\%$ \\
\hline \multirow[t]{9}{*}{ Construtores } & Bol & 48 & 17,9 & 153 & 57,1 & 17 & 6,3 & 40 & 14,9 \\
\hline & Caça & 6 & 2,2 & 22 & 8,2 & 38 & 14,2 & 177 & 66,0 \\
\hline & Galinha & 48 & 17,9 & 158 & 59,0 & 25 & 9,3 & 30 & 11.2 \\
\hline & Jabá & 17 & 6.3 & 2 & 0.7 & 0 & 0 & 5 & 1,9 \\
\hline & Peixe & 82 & 30,6 & 135 & 50,4 & 20 & 7,5 & 30 & 11.2 \\
\hline & Ovos & 66 & 24,6 & 102 & 38,1 & 13 & 4,9 & 63 & 23,5 \\
\hline & Lelte em pó & 170 & 63,4 & 28 & 10,4 & 5 & 1,9 & 33 & 12,3 \\
\hline & Queijo & 24 & 9.0 & 45 & 16,8 & 26 & 10,1 & 152 & 56.7 \\
\hline & Feijẫo & 1 & 0.4 & 36 & 13,4 & 6 & 2,2 & 7 & 2,6 \\
\hline Alimentos & Pâo & 0 & 0 & 37 & 13,8 & 2 & 0,7 & 38 & 14,2 \\
\hline \multirow[t]{6}{*}{ Energé ticos } & Arroz & 253 & 94,4 & 10 & 3.7 & 1 & 0,4 & 4 & 1,5 \\
\hline & Milho & 6 & 2,2 & 29 & 10,8 & 14 & 5,2 & 212 & 79.5 \\
\hline & Bolacha & 123 & 45,9 & 73 & 27,2 & 12 & 4,5 & 37 & 13,8 \\
\hline & Macarrâo & 1 & 0,4 & 29 & 10,8 & 0 & 0 & 0 & 0 \\
\hline & $\left({ }^{*}\right)$ & $58+$ & 21,6 & 1 & 0,4 & 0 & 0 & 0 & 0 \\
\hline & Óleo Vegetal & 244 & 91,0 & 6 & 2,2 & 0 & 0 & 8 & 3.0 \\
\hline Alimentos & $(*)$ & 213 & 79,5 & 18 & 6,7 & 0 & 0 & 29 & 10.8 \\
\hline Reguladores & $(* \cdots)$ & 155 & 57,8 & 64 & 23,9 & 8 & 3,0 & 38 & 14,2 \\
\hline \multirow[t]{3}{*}{ Bebidas } & Café & 170 & 63,4 & 0 & 0 & 0 & 0 & 0 & 0 \\
\hline & Refrigerantes & 45 & 16,8 & 18 & 6,3 & 0 & 0 & 5 & 1,9 \\
\hline & Suco & 10 & 3,7 & 0 & 0 & 0 & 0 & 0 & 0 \\
\hline
\end{tabular}

$\left(^{*}\right)=$ Farinha de mandioca e batata.

$(* *)=$ Couve, tomate, cebola, pimentăo, cheiro-verde, cenoura, alho e maxixe.

$(* *)=$ Banana, laranja, abacate, cajú, manga, açaí e melancia. 
falciparum).

\section{Inquérito Alimentar}

A dieta se caracterizou por uma alta freqüência de consumo diário de alimentos do grupo energético, sendo os principais: arroz $(94,4 \%)$, óleo vegetal $(91,0 \%)$ e bolacha $(45,9 \%)$. Registrou-se também alta porcentagem de alimentos do grupo dos construtores, tais como o leite em pó $(63,4 \%)$, peixe $(30,6 \%)$, ovos $(24,6 \%)$, carne de galinha e de boi $(17,9 \%)$. Do mesmo modo, evidenciase a utilização de alimentos reguladores $(79,5 \%)$, sendo os de maior uso para tempero dos alimentos: couve, tomate, cebola, pimentão, cheiro verde, cenoura e alho. Ainda dentro deste grupo, registramos o consumo de frutas $(57,8 \%)$, sendo as de maior preferência: banana, laranja e abacate.

\section{DISCUSSÃO}

Considerando a importância epidemiológica da malária na região Amazônica, bem como a ausência de informações a respeito do comprometimento do estado nutricional do Amazônida em decorrência da infecção malárica, o presente estudo procurou avaliar o perfil nutricional de portadores de malária, inicialmente, por meio das variáveis antropométricas. Estas medidas estimam qualitativamente, as variações nas dimensões físicas da composição corporal, e, estão sendo cada vez mais utilizadas em estudos epidemiológicos, de avaliação das condições de saúde e nutrição de individuos e de grupos populacionais (Jelliffe, 1968; Gorstein, 1990, Vasconcelos, 1995).

A análise dos resultados da presente pesquisa mostra que a população estudada, apesar de constituída de portadores de malária, apresentou valores médios do IMC e CBM, dentro dos padrões de normalidade, tanto para os pacientes com malária causada pelo $P$. vivax quanto por $P$. falciparum. No entanto, o valor médio da PCT, mostrou-se abaixo dos limites considerados de normalidade, independentemente, do tipo de Plasmodium.

Estes resultados são conflitantes com as informações da literatura que referem um perfeito mecanismo de dependência resultante da interação do binômio infecção/nutrição, cujo equilibrio, vai mediar o sentido de utilização dos combustiveis metabólicos. Divergem também, do consenso de que cada tipo de Plasmodium, possui características especificas que determinarão a patogenia da doença no homem, e, a magnitude do seu quadro clínico. Portanto, em resposta a infecção malárica, podem ocorrer perdas de reservas orgânicas, devido em parte a anorexia, vômitos, e ao efeito do estado hipercatabólico associado à febre. Deste modo, estas alteraçôes contribuem para acelerar o consumo das reservas calóricas do organismo, armazenadas como gordura, principalmente, sob a forma de tecido adiposo (Beisel, 1980; McGregor, 1982; Berezin \& Succi, 1989; Delgado et al., 1995; Pereira et al., 1995). 
A possível explicação para o comportamento anômalo das variáveis antropométricas verificado no presente estudo, seria o fato de que a grande maioria dos pacientes investigados, era constituida de adultos jovens $(81,3 \%)$, do sexo masculino $(73,9 \%)$, e, que destes $71,6 \%$ estavam vinculados à categoria de trabalhadores com atividade braçal e agricola, o que certamente exige, em sua jornada diária de trabalho, uma freqüência de esforço físico de alta intensidade, que acarreta maior desenvolvimento muscular e cuja hipertrofia, se refletiu nas dimensões físicas do organismo.

A avaliação do estado nutricional da população estudada, foi ainda complementada pela investigação hematológica e bioquímica. Estas medidas são mais objetivas e fornecem informação mais direta do estado nutricional, sendo de reconhecida eficácia para diagnosticar precocemente, alterações metabólicas e fisiológicas do organismo, decorrentes de processos patológicos: absorção defeituosa, insuficiência dietética ou excesso de consumo alimentar. Os resultados desta investigação, mostrou que os portadores de malária não apresentavam alterações nas concentrações médias de hematócrito, hemoglobina, glicose, ferro sérico e triglicerídeos. No entanto, as concentrações médias de proteína total e HDL colesterol, encontravam-se no limite inferior de normalidade $(6,9$ $\mathrm{g} / \mathrm{dL}$ e $29,9 \mathrm{mg} / \mathrm{dL}$ ) respectivamente. Deve ser enfatizado que o inquérito de consumo alimentar, revelou que a população estudada teve acesso a uma dieta bem estruturada, sendo freqüente o consumo de alimentos do grupo dos construtores (peixe, carne e ovos), abundante nos energéticos (arroz e pão), gordura vegetal (óleos), e, em menor quantidade, os reguladores (verduras e frutos). Do ponto de vista endócrino, o estado nutricional do indivíduo expressa uma função interativa entre o consumo de alimentos e aproveitamento biológico, sendo o nivel de utilização orgânica dos nutrientes, dependente de condicionantes físiológicos, e, sobretudo, patológicos, que alteram a ingestão, absorção e metabolismo. No nível ambiental, foram registrados como fatores agravantes do estado nutricional da população estudada, além da malária, alto índice de parasitose gastrointestinal $(72,7 \%)$, predominando o poliparasitismo $(62,5 \%)$, precárias condições de habitação (casas de madeiras 63,4\%); baixo nível de escolaridade $65,9 \%$, baixo poder aquisitivo, etilismo $50,0 \%$ e tabagismo $34,3 \%$. Curiosamente, e, apesar da presença constante de todos estes fatores agravantes, não foram registrados na população estudada, alterações significativas nos valores dos indicadores antropométricos, hematológicos e bioquímicos.

Neste sentido, deve ser levado em consideração, o fato do universo estudado ter sido constituido, em sua totalidade, por pacientes que procuravam a FNS, para esclarecimento diagnóstico do processo febril inicial, portanto, no 
estágio inicial da malária, quando ainda não é possivel detectar alterações metabólicas nos líquidos e estoques corporais. Do mesmo modo, é perfeitamente possivel se admitir que a dieta consumida pelos portadores de malária (rica em carboidratos e proteínas), tenha sido um fator atenuante, a impedir a depleção dos nutrientes circulantes $\mathrm{e}$ estoques corporais, decorrentes do catabolismo celular, provocado pela presença do Plasmodium, e, atúação dos demais fatores agravantes do estado nutricional da referida população.

\section{AGRADECIMENTOS}

Ao $\mathrm{CNPq}$ pela ajuda financeira ao desenvolvimento desta pesquisa. (Processo ${ }^{0}$ 000400356/97).

\section{Bibliografia citada}

Albuquerque, B.C.J.; Mutis, M.C.S. 1998. A Malária no Amazonas. In: Rojas L.B.I.; Toledo, L.M. (Org.). Espaço e Doença: Um Olhar sobre o Amazonas. Rio de Janeiro: Ed. Fiocruz. p.11.2.1 - 11.2.10.

Alecrim, J.K.C. 1995. Os Impactos Econômicos da Malária na Cidade de Manaus. Monografia para obtenção do Grau de Economista. Universidade Federal do Amazonas. Manaus. 22p.

Alencar, F.H. 1988 Avaliação do Estado Nutricional de Crianças Pré-escolares residentes na Area Urbana do Municipio de Anhembi. Tese de Doutorado. Universidade Estadual Paulista,. São Paulo. $128 \mathrm{p}$.

Batista, R.S. 1995. Nutrição e Saúde, Novas Perspectivas. JBM. Rio de Janeiro, 69, (2): 98-101.

Beisel, W.R 1980. Effects of infection on nutricional status and immunity. Fed. Proc. 39 (13): 3105 -3108.
Berezin, E.N.; Succi, R.C.1989. DesnutriçãoImunidade e Infecção. Ped. Mod. São Paulo, 24 (3): 81-85.

Bucolo, G.; David, H. 1973. Quantitative determination of serum triglycerides by the use of enzymes. Clin. Chem. Boston, 19 (5): 476- 482 .

Dean, A.G.; Dean, J.A.; Burton, A.H.; Dicker, R.C. 1990. Epi Info, versión 5.01: a word precessing, database, and statistics program for epidemiology on micro-computers. Centers for Disease Control, Atlanta, Georgia, USA, 368p.

Delgado, L.F.; Fisberg, M.; Carvalho, E.S. 1995. Infecção e desnutrição. Ped. Mod. ( Ediç. Espec.) São Paulo, 31: 633- 642.

Ferraroni, J.J.; Speer, C.A. 1981. Aspectos imunoepidemiológicos da infecção pôr Plasmodium e perspectivas de erradicação da malária humana. Ciência e Cultura (Curitiba), 33: 362-368.

Frisancho, A.R. 1974. Triceps skin fold and upper arm muscle size norms for assessment of nutritional status. Am. J, Clin. Nutr. Bethesda - USA, 27 (7-12): 1052 1058.

Gorstein, J. 1990 Evaluación del estado de nutrición. La clasificación de la desnutrición veria según el método usado para determinar la edad. Bol. Sanit Panam. Ginebra.108 (1); 27-38.

Goodwin, J.F.; Murphy, B.; Guillmette, M. 1966. Direct Measurement of Serum Iron and Binding Capacity. Clin. Chem. Boston, 12(2): 47-57.

Izzo, C,; Grillo, F.; Murador, E. 1981, Improved Method for Determination of High-Density-Lipoprotein Cholesterol. I. Isolation of High-Density Lipoproteins by Use of Polyethlene Glycol 6000. Clin. Chem. Boston, 27 (3): 371-374.

Jelliffe, D.B. 1968. Evalución del Estado de Nutrición de la Comunidad; com especial referencia a las encuestas en las regiones en desarrollo. Ginebra: Org. Mundial de la Salud. . $291 \mathrm{p}$.

McGregor, I.A.1982. Malaria: Nutritional Implications. Rev. Inf. Dis. Chicago, 4 (4): 
$798-804$.

Organizacion Mundial De La Salud-OMS 1993. Conferencia Ministerial sobre el Paludismo. Bol. Sanit. Panam., Washington, 113 (5/6): 503-506.

Organización Panamericana De La SaludOPAS 1992. La salud mediante la nutricion. Bol. Sanit. Panam., Washington, 113 (5/6): 502- 506.

Organización Panamericana De La Salud OPAS 1996. Situación de la malaria en las Americas. Bol. Epidem., Washington, 17(4):1-6.

Paiva, S.A.R.; Campana, A.O. 1992, O diagnóstico da Desnutrição EnergéticoProtéica em Pacientes Adultos: Antropometria. Cad. Nutri. São Paulo, 4: 27-37

Pereira, P.C.M.; Meira, D.A.; Duri, P.R.; Souza, N.; Burini, R.C. 1995. The malarial impact on the nutritional status of amazonian adult subjects. Rev. Inst. Med. Trop. São Paulo, 37 (1): 19-24.

Shrimpton, R.; Giugliano, R. 1979. Consumo de alimentos e alguns nutrientes em Manaus. Amazonas. Acta Amazonica. Manaus-Am, 9 (1): 117-141.

Souza, J.M.; Couto, A.A.R.; Da Silva, E.B.; Adbdon, N.P.; Da Silva, R.S.U. 1997. Malảria. In: Leão, R.N.Q. Doenças Infecciosas e Parasitárias - Enfoque Amazônico. Belém: Ed. CEJUP, p. 645669.
Tauil, P.L. 1996. Epidemiologia. In: Veronesi, R.; Focaccia, R. Tratado de Infectologia. São Paulo: Atheneu. p. 1264-1268.

Thomas, A.E.; McKay, D.A.; Cutlip, M.B. 1976. A normograph method for assessing body weight. Am. J. Clin. BethesdaUSA, 29: 302 - 304

Trinder, R. 1969 Glicose Enzimática. Ann. Clin. Biochem. 6(4): 538-544.

Van Kampen, E.J.; Zijstra, W.G. 1961. Standardization of hemoglobinometry. II. The hemiglobincyanide method. Clin. Chim. Acta. 6 (4): 538- 544.

Vasconcelos, F.A.G. 1995. Avaliação Nutricional de Coletividades: Textos de Apoio didático. $2^{\mathrm{a}}$ ed. Florianópolis: Edit. da UFSC, 154p.

Wilkinson, L. 1990. Systat: The System for Statistics. SYSTAT INC., Evanston. Illinois.

Yuyama, L.K.O.; Cozzolino, S.M.F. 1995. Determinação dos Teores de $\mathrm{Zn}, \mathrm{Ca}, \mathrm{Se}$, $\mathrm{Cu}, \mathrm{K} . \mathrm{MG}$, na Dieta Regional de Manaus, Am. Rev. Inst. Adolfo Lutz. São Paulo, 55 (1): 45-50. 